\title{
Interference of Bose-Einstein condensates split with an atom chip
}

\author{
Y. Shin, C. Sanner, G.-B. Jo, T. A. Pasquini, M. Saba, W. Ketterle, and D. E. Pritchard* \\ MIT-Harvard Center for Ultracold Atoms, Research Laboratory of Electronics, Department of Physics, \\ Massachusetts Institute of Technology, Cambridge, Massachusetts 02139, USA \\ M. Vengalattore and M. Prentiss \\ MIT-Harvard Center for Ultracold Atoms, Jefferson Laboratory, Physics Department, Harvard University, \\ Cambridge, Massachusetts 02138, USA
}

(Received 10 June 2005; published 11 August 2005)

\begin{abstract}
We have used a microfabricated atom chip to split a single Bose-Einstein condensate of sodium atoms into two spatially separated condensates. Dynamical splitting was achieved by deforming the trap along the tightly confining direction into a purely magnetic double-well potential. We observed the matter wave interference pattern formed upon releasing the condensates from the microtraps. The intrinsic features of the quartic potential at the merge point, such as zero trap frequency and extremely high field-sensitivity, caused random variations of the relative phase between the two split condensates. Moreover, the perturbation from the abrupt change of the trapping potential during the splitting was observed to induce vortices.
\end{abstract}

DOI: 10.1103/PhysRevA.72.021604

PACS number(s): 03.75.Dg, 03.75.Kk, 39.20.+q

Coherent manipulation of matter waves is the ultimate goal of atom optics, and diverse atom optical elements have been developed such as mirrors, beamsplitters, gratings, and waveguides. An atom chip integrates these elements on a microfabricated device allowing precise and stable alignment [1-3]. Recently, this atom chip technology has been combined with Bose-Einstein condensed atoms [4,5], and opened the prospect for chip-based atom interferometers with BoseEinstein condensates. Despite various technical problems [6-10], there have been advances toward that goal, such as excitationless propagation in a waveguide [6] and demonstration of a Michelson interferometer involving splitting along the axis of a single waveguide [11].

Coherent splitting of matter waves into spatially separate atomic wave packets with a well-defined relative phase is a prerequisite for further applications such as atom interferometry and quantum information processing, and it has been a major experimental challenge. The methods envisioned for coherent splitting on atom chips can be divided in two classes. One is splitting in momentum space and subsequently generating a spatial separation, using scattering of atoms from a periodic optical potential $[11,12]$. The other is dynamical splitting by directly deforming a single wave packet into two spatially separated wave packets, which can be considered as cutting off the link between two wave packets, i.e., stopping tunneling through the barrier separating two wave packets. Splitting in momentum space has led to remarkably clean interferometric measurements when the atoms were allowed to propagate freely after splitting, but it has been pointed out that momentum splitting of confined atoms (e.g., inside a waveguide) is problematic due to spatially dependent phase shifts induced by atomatom interactions during separation $[11,13]$. Dynamical splitting in real space instead is perfectly compatible with

*URL: http://cua.mit.edu/ketterle_group/ keeping atoms confined, a feature beneficial to the versatility of interferometers. There has been a theoretical debate concerning the adiabatic condition for coherent dynamical splitting [14-17]. In our recent experiment with an optical double-well potential, we demonstrated that it is possible to dynamically split a condensate into two parts in a coherent way [18].

In this work, we studied the dynamical splitting of condensates in a purely magnetic double-well potential on an atom chip. We developed an atom chip to generate a symmetric double-well potential and succeeded in observing the matter wave interference of two split condensates, from which the coherence of the splitting process was investigated. We found that the mechanical perturbations during splitting are violent enough to generate vortices in condensates. We discuss the adiabatic condition of the splitting process.

A magnetic double-well potential was realized with an atom chip using a two-wire scheme [19]. The experimental setup of the atom chip is shown in Fig. 1. When two chip wires have currents, $I_{C}$, in the $-y$ direction and the external magnetic field, $B_{x}$, is applied in the $+x$ direction, two lines of local minima in the magnetic field are generated above the chip surface. Each local minimum has a quadruple field configuration in the $x z$ plane, and with an additional nonzero magnetic field in the axial direction ( $y$-direction), two Ioffe-Pritchard magnetic traps can be formed. The relative magnitude of $B_{x}$ to the field from $I_{C}$ determines the direction of separation and the distance of the two traps. The atom chip was set to face downward and the two traps are vertically (horizontally) separated when $B_{x}<B_{x 0}\left(B_{x}>B_{x 0}\right) . \quad B_{x 0}=\mu_{0} I_{C} / \pi d$ is the critical field magnitude for merging two magnetic harmonic potentials to form a single quartic potential, where $d$ is the distance between the two chip wires and $\mu_{0}$ is the permeability of the vacuum. The merge point is located at the middle of the two wires and $d / 2$ away from the chip surface. In our experiment, $d=300 \mu \mathrm{m}$; thus, the splitting happened 


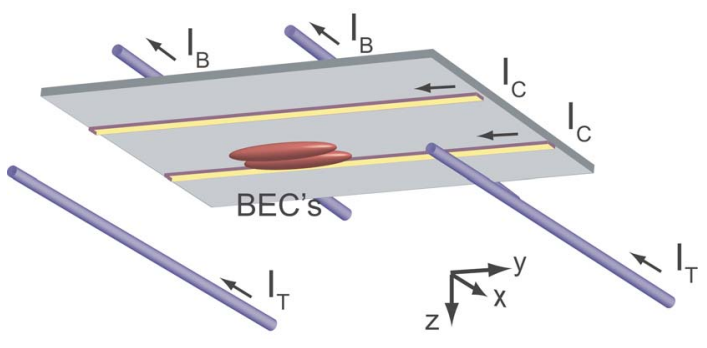

FIG. 1. (Color online) Schematic diagram of the atom chip. A magnetic double-well potential was created by two chip wires with a current $I_{C}$ in conjunction with an external magnetic field. The distance between the two chip wires was $300 \mu \mathrm{m}$. A pair of external wires with $I_{B}$ provided the axial confinement along the $y$ direction, and another pair of external wires with $I_{T}$ were used for reducing the antisymmetry effect. (For details, see text.) Gravity was in the $+z$ direction.

more than $200 \mu \mathrm{m}$ away from the chip wires to avoid deleterious surface effects [6-10]. The chip wires of $12 \mu \mathrm{m}$ height and $50 \mu \mathrm{m}$ width were electroplated with $\mathrm{Au}$ on a thermally oxidized Si substrate with a $2-\mu \mathrm{m}$-thick Au evaporated film. The chip was glued on an Al block for heat dissipation [20] and the current capacity was $5 \mathrm{~A}$ in a continuous mode.

The axial trapping potential was carefully designed to ensure that condensates split perpendicular to the axial direction and stay in the same axial position. The two wells have opposite responses to $B_{z}$ : positive $B_{z}$ makes the left (right) well move upward (downward). If $B_{z}$ changes along the axial direction, the two wells are no longer parallel and the gravitational force would cause an axial displacement of the two split condensates. When endcap wires are placed only on the chip surface as in our previous work [21], a nonzero field gradient $\partial B_{z} / \partial y$ inevitably accompanies a field curvature $\partial^{2} B_{y} / \partial y^{2}$ for the axial confinement, i.e., $B_{z}$ changes from positive to negative along the axial direction. In order to provide the axial confinement and at the same time minimize $\partial B_{z} / \partial y$, we placed two pairs of external wires $1.5 \mathrm{~mm}$ above and $4 \mathrm{~mm}$ below the chip surface. This three-dimensional design of axial confinement was necessary for obtaining the interference signal of two split condensates. Moreover, maintaining the geometric symmetry of two wells will be crucial for longer coherence time after splitting [18].

The splitting process was demonstrated with the experimental procedures described in Fig. 2. Bose-Einstein condensates of $\left|F=1, m_{F}=-1\right\rangle{ }^{23} \mathrm{Na}$ atoms were transferred and loaded in a magnetic trap generated by the atom chip $[6,21,22]$. Experimental parameters were $I_{C}=1.8 \mathrm{~A}, B_{x 0}$ $=24 \mathrm{G}, B_{y}=1 \mathrm{G}$, and the axial trap frequency $f_{y}=13 \mathrm{~Hz}$. Condensates were first loaded in the bottom well, $500 \mu \mathrm{m}$ away from the chip surface, brought up to $30 \mu \mathrm{m}$ below the merge point in $1 \mathrm{~s}$, and held there for $2 \mathrm{~s}$ to damp out excitations. The long-living axial dipole excitation induced in the transfer phase was damped by applying a repulsive potential wall at the one end of the condensates with a blue-detuned
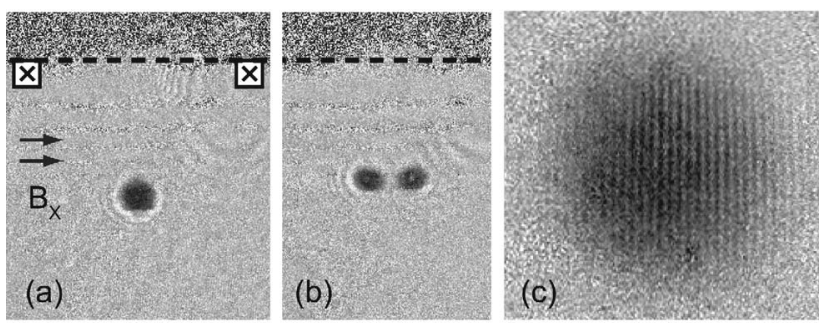

FIG. 2. Splitting of condensates. (a) Condensates were initially loaded and prepared in the bottom well and (b) split into two parts by increasing the external magnetic field, $B_{x}$. For clarity, two condensates were split by $80 \mu \mathrm{m}$. The dash line indicates the chip surface position. The currents in the chip wires flow into the page and $B_{x}$ is parallel to the wire separation. Two condensates were released from the magnetic double-well potential and the matter wave interference pattern of two condensates formed after time-offlight. (c) Typical absorption image of interference fringes taken after $22 \mathrm{~ms}$ time-of-flight. The fringe spacing was $14.8 \mu \mathrm{m}$, corresponding to a condensate separation of $25.8 \mu \mathrm{m}$.

laser beam $(532 \mathrm{~nm})^{1}$. The whole procedure was carried out with a radio-frequency (rf) shield and, just before splitting, condensates contained over $8.0 \times 10^{5}$ atoms without a discernible thermal population. Splitting was done by ramping $\Delta B_{x}=B_{x}-B_{x 0}$ linearly from $-140 \mathrm{mG}$ to $100 \pm 20 \mathrm{mG}$ in $200 \mathrm{~ms}$. The separation between two condensates was controlled by the final value of $B_{x}$. The magnetic trap was then quickly turned off within $20 \mu \mathrm{s}$, a duration much shorter than the inverse of any trap frequency, preventing random perturbations. High-contrast matter wave interference fringes were observed after releasing the condensates and letting them expand in time-of-flight (Fig. 2), indicating that the splitting procedure was smooth enough to produce two condensates having uniform phases along their long axial axis perpendicular to the splitting direction. In order to study the coherence of the splitting, the relative phase of the two split condensates was determined from the spatial phase of the matter wave interference pattern.

The relative phase of two split condensates turned out to be unpredictable when they were fully separated (Fig. 3). The separation of two condensates was determined from the spacing, $\lambda_{s}$, of the interference fringes, using the formula $d$ $=h t / m \lambda_{s}$ where $h$ is Planck's constant, $m$ is atomic mass, and $t$ is time-of-flight. The typical fringe spacing was $\lambda$ $\approx 15 \mu \mathrm{m}$ with $t=22 \mathrm{~ms}$, corresponding to $d \approx 26 \mu \mathrm{m}$. Given the precise knowledge of the fabricated wires, the full trap parameters can be calculated. Assuming that the condensates followed trap centers in the motional ground state, it was found that when the barrier height was over $1.5 \mathrm{kHz}$, the

\footnotetext{
${ }^{1}$ In a perfectly symmetric double-well potential, two condensates would oscillate in phase after splitting. Furthermore, this could be used for developing a rotation-sensitive atom interferometer with a guiding potential. However, the axial trap frequencies for the two wells were found to be different by $12 \%$ due to the imperfect fabrication of wires.
} 


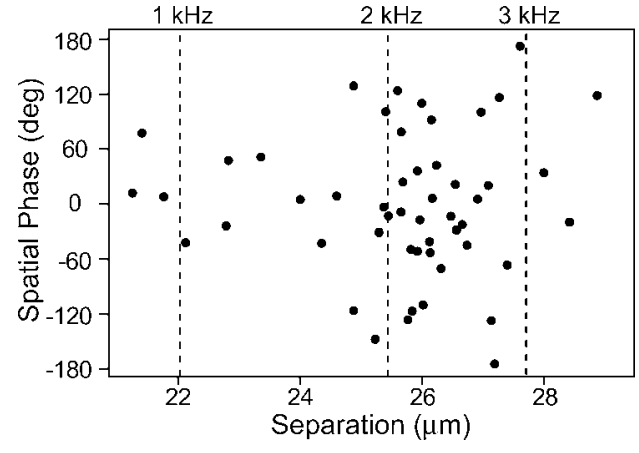

FIG. 3. Spatial phase of interference fringes. The separation of two condensates was determined from the spacing of interference fringes. Fifty repetitions of the same experiment are plotted, where the experimental control value for the external magnetic field, $B_{x}$, was fixed when the atoms were released. Three dash lines indicate the separations of two wells with the barrier height of $1 \mathrm{kHz}$, $2 \mathrm{kHz}$, and $3 \mathrm{kHz}$, respectively.

relative phase started to be random. ${ }^{2}$ Since the chemical potential of the condensates, $\mu=1.4 \pm 0.2 \mathrm{kHz}$, was very close to this barrier height, the condensates just started to lose their coupling at this point.

Surprisingly, a phase singularity was observed in the interference patterns with high visibility. The fork shape of interference fringes represents a phase winding around a vortex core [23]. This vortex interference pattern appeared more frequently with faster splitting and further separation. An external perturbation can lead to internal excitations in condensates. Splitting might be considered as slicing condensates in two parts. The fact that the observed "forks" (Fig. 4) always open towards the top implies that the slicing always occurred in the same direction and created either vortices with positive charge on the left side or with negative charge on the right side. A possible vortex formation mechanism is topological imprinting when the zero point of the magnetic field crosses though condensates resulting in a doubly quantized vortex in spin-1 condensates [21,22]. However, since we have never observed the interference pattern of a doubly quantized vortex, we think that this scenario is unlikely.

We now discuss how the trapping potential changes during the splitting process (Fig. 5). When condensates split into two wells, the trap frequency, $f_{x}$, in the splitting direction vanishes and the separation of two wells abruptly increases to $15 \mu \mathrm{m}$ with a small magnetic field change of $\delta B_{x} \approx 10 \mathrm{mG}$. For a single particle in a harmonic potential, the quantity $\alpha \equiv\left(1 / f_{x}^{2}\right)\left(\partial f_{x} / \partial B_{x}\right)\left(d B_{x} / d t\right)$ accounts for the transition probability from the ground state to the first excited state and parametrizes the external adiabaticity of the process, neglecting the collective excitations of a condensate. $\alpha \ll 1$ should be maintained to keep condensates staying in the motional ground state. With $d B_{x} / d t=1.2 \mathrm{G} / \mathrm{s}, \alpha<1$ at $f_{x}>150 \mathrm{~Hz}$, but obviously, $\alpha$ diverges to infinity near the merge point and its definition no longer holds. Since the

\footnotetext{
${ }^{2}$ When the separation was less than $20 \mu \mathrm{m}$ and two condensates were linked, the uncertainty of the spatial phase of fringes was less than $60^{\circ}$.
}
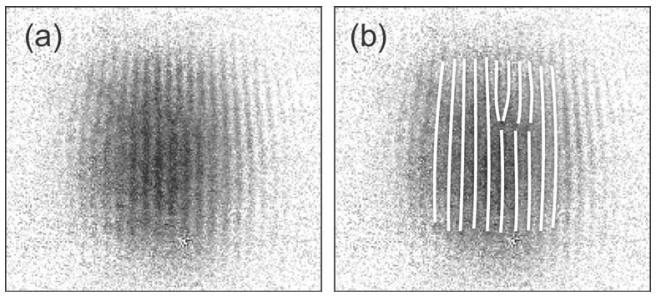

FIG. 4. Vortex interference. (a) An absorption image showing the vortex interference pattern of a vortex state. The probability of vortex generation was $\sim 8 \%$ for the experimental parameters of Fig. 3 , where data points with vortices were not included. Vortex interference patterns appeared more frequently with faster splitting and further separation. (b) Same as (a), but with lines indicating regions with constant phase.

energy level spacing diminishes, the adiabatic condition in the quartic potential around the merge point becomes more stringent. The abrupt change of trapping potential will induce mechanical perturbations of condensates. Subsequent dissipation or coupling into internal excitation modes [24] would make the relative phase of two split condensates unpredictable. The observed phase singularity definitely shows the breakdown of adiabaticity.

One possible alternative to avoid passing through the merge point is starting with two weakly linked condensates in a double-well potential where the barrier height is lower than the chemical potential of condensates and controlling the coupling between two condensates with a small change of the barrier height. This method was used to reduce the motional perturbation in our previous work [18]. However,
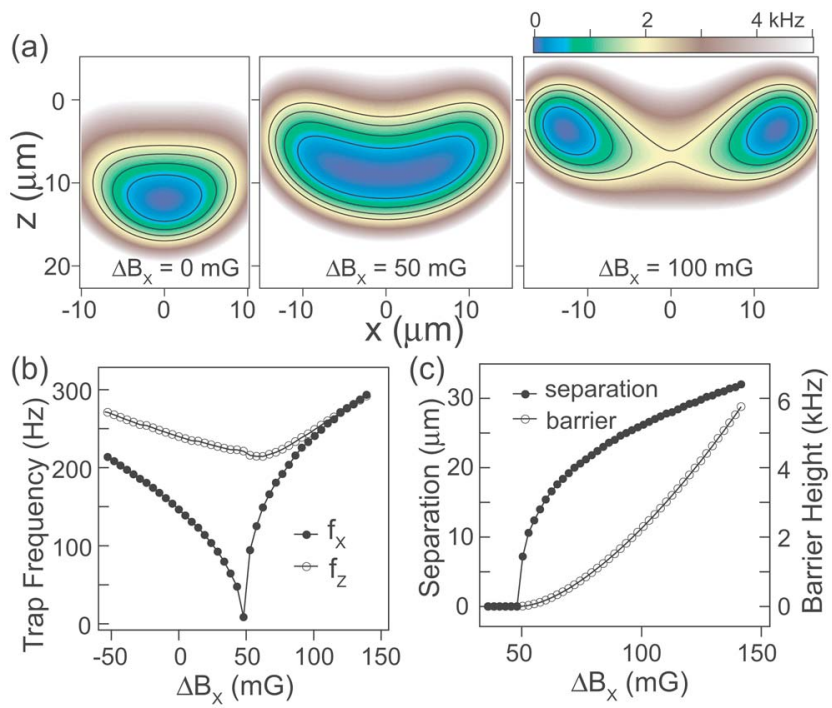

FIG. 5. (Color online) Trapping potential during splitting. (a) Radial cross sections of trapping potential including gravity for $\Delta B_{x}=0,50$, and $100 \mathrm{mG}$, where $\Delta B_{x}$ is the field deviation from the critical field magnitude $B_{x 0}$ which is the field magnitude for forming a single quartic trap. The origin of coordinates is the merge point without gravity. Contour lines correspond to $0.5,1,1.5$, and $2 \mathrm{kHz}$ above the bottom of the trap. (b) Trap frequencies in each direction. (c) Separation of two trap centers and barrier height between two wells. 
since the sensitivity of the trapping potential to the magnetic field is extremely high when the trap centers are close to the merge point, it was technically difficult to have a stable double-well potential with a small barrier height. The lifetime of condensates measured around the merge point was $>5 \mathrm{~s}$ away from the merge point $\left(\Delta B_{x}<-50 \mathrm{mG}\right.$ or $\Delta B_{x}$ $>150 \mathrm{mG})$ and $<100 \mathrm{~ms}$ near the merge point $\left(0<\Delta B_{x}\right.$ $<100 \mathrm{mG}){ }^{3}$ With a barrier height of $0.5 \mathrm{kHz}$ in our experiment, the sensitivity of the barrier height and the condensate separation to $B_{x}$ is $0.04 \mathrm{kHz} / \mathrm{mG}$ and $0.3 \mu \mathrm{m} / \mathrm{mG}$, respectively. $\delta B_{x}=1 \mathrm{mG}$ corresponds to $\delta I_{C}=7.5 \times 10^{-5} \mathrm{~A}$. Extreme current stabilization and shielding of ambient magnetic field fluctuations may be necessary for controlling a phasecoherent splitting process. Another alternative for preparing a coherent state of two spatially separated condensates is first preparing two condensates in the ground states in

${ }^{3}$ For positions with $\Delta B_{x}>0$ ("after" splitting), the condensates were moved to the left well without passing through the merge point. each well and then establishing a well-defined relative phase with an optical method [25]. This scheme is currently under investigation.

In conclusion, we have demonstrated the interference of two Bose-Einstein condensates released from an atom chip. The condensates were created by dynamical splitting of a single condensate and could be kept confined in a magnetic double-well potential, separated by an arbitrary distance. We studied the coherence of the dynamical splitting process by measuring the relative phase of two split condensates and identified technical limitations, intrinsic to the magnetic field geometry, that prevented coherent splitting with a predictable phase. This study is a promising step in the route towards atom chip interferometers and might serve as a guide for the design of future microfabricated atom optics devices.

This work was funded by ARO, NSF, ONR, DARPA, and NASA. C.S. acknowledges additional support from the Studienstiftung des deutschen Volkes, G.-B.J. from the Samsung Lee Kun Hee Scholarship Foundation, and M.S. from the Swiss National Science Foundation.
[1] D. Müller, D. Z. Anderson, R. J. Grow, P. D. D. Schwindt, and E. A. Cornell, Phys. Rev. Lett. 83, 5194 (1999).

[2] N. H. Dekker, C. S. Lee, V. Lorent, J. H. Thywissen, S. P. Smith, M. Drndić, R. M. Westervelt, and M. Prentiss, Phys. Rev. Lett. 84, 1124 (2000).

[3] R. Folman, P. Krüger, D. Cassettari, B. Hessmo, T. Maier, and J. Schmiedmayer, Phys. Rev. Lett. 84, 4749 (2000).

[4] H. Ott, J. Fortagh, G. Schlotterbeck, A. Grossmann, and C. Zimmermann, Phys. Rev. Lett. 87, 230401 (2001).

[5] W. Hänsel, P. Hommelhoff, T. W. Hänsch, and J. Reichel, Nature (London) 413, 498 (2001).

[6] A. E. Leanhardt, A. P. Chikkatur, D. Kielpinski, Y. Shin, T. L. Gustavson, W. Ketterle, and D. E. Pritchard, Phys. Rev. Lett. 89, 040401 (2002).

[7] J. Fortágh, H. Ott, S. Kraft, A. Günther, and C. Zimmermann, Phys. Rev. A 66, 041604(R) (2002).

[8] A. E. Leanhardt, Y. Shin, A. P. Chikkatur, D. Kielpinski, W. Ketterle, and D. E. Pritchard, Phys. Rev. Lett. 90, 100404 (2003).

[9] M. P. A. Jones, C. J. Vale, D. Sahagun, B. V. Hall, and E. A. Hinds, Phys. Rev. Lett. 91, 080401 (2003).

[10] J. Estève, C. Aussibal, T. Schumm, C. Figl, D. Mailly, I. Bouchoule, C. I. Westbrook, and A. Aspect, Phys. Rev. A 70, 043629 (2004).

[11] Y.-J. Wang, D. Z. Anderson, V. M. Bright, E. A. Cornell, Q. Diot, T. Kishimoto, M. Prentiss, R. A. Saravanan, S. R. Segal, and S. Wu, Phys. Rev. Lett. 94, 090405 (2005).

[12] P. J. Martin, B. G. Oldaker, A. H. Miklich, and D. E. Pritchard, Phys. Rev. Lett. 60, 515 (1988).
[13] M. Olshanii and V. Dunjko, e-print cond-mat/0505358.

[14] J. Javanainen and M. Wilkens, Phys. Rev. Lett. 78, 4675 (1997).

[15] A. J. Leggett and F. Sols, Phys. Rev. Lett. 81, 1344 (1998).

[16] C. Menotti, J. R. Anglin, J. I. Cirac, and P. Zoller, Phys. Rev. A 63, 023601 (2001).

[17] L. Pezzé, A. Smerzi, G. P. Berman, A. R. Bishop, and L. A. Collins, e-print cond-mat/0411567.

[18] Y. Shin, M. Saba, T. A. Pasquini, W. Ketterle, D. E. Pritchard, and A. E. Leanhardt, Phys. Rev. Lett. 92, 050405 (2004).

[19] E. A. Hinds, C. J. Vale, and M. G. Boshier, Phys. Rev. Lett. 86, 1462 (2001).

[20] S. Groth, P. Krüger, S. Wildermuth, R. Folman, T. Fernholz, J. Schmiedmayer, M. Mahalu, and I. Bar-Joseph, Appl. Phys. Lett. 85, 2980 (2004).

[21] Y. Shin, M. Saba, M. Vengalattore, T. A. Pasquini, C. Sanner, A. E. Leanhardt, M. Prentiss, D. E. Pritchard, and W. Ketterle, Phys. Rev. Lett. 93, 160406 (2004).

[22] A. E. Leanhardt, A. Görlitz, A. P. Chikkatur, D. Kielpinski, Y. Shin, D. E. Pritchard, and W. Ketterle, Phys. Rev. Lett. 89, 190403 (2002).

[23] S. Inouye, S. Gupta, T. Rosenband, A. P. Chikkatur, A. Görlitz, T. L. Gustavson, A. E. Leanhardt, D. E. Pritchard, and W. Ketterle, Phys. Rev. Lett. 87, 080402 (2001).

[24] H. Ott, J. Fortágh, S. Kraft, A. Günther, D. Komma, and C. Zimmermann, Phys. Rev. Lett. 91, 040402 (2003).

[25] M. Saba, T. A. Pasquini, C. Sanner, Y. Shin, W. Ketterle, and D. E. Pritchard, Science 307, 1945 (2005). 